\title{
Autoestima y procrastinación en el ámbito académico: un meta-análisis
}

\section{Self-esteem and procrastination in the academic field: a meta-analysis}

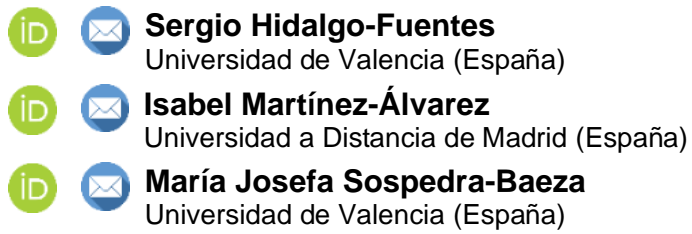

\section{Resumen}

La procrastinación es un comportamiento común en el ámbito académico asociado a diversas consecuencias negativas. La autoestima, entendida como una actitud global hacia uno mismo, es una de las variables que se ha asociado con la procrastinación académica. El objetivo del presente trabajo es examinar mediante técnicas meta-analíticas la relación entre la autoestima y la procrastinación en estudiantes. Se realizó una búsqueda bibliográfica en las bases de datos Psyclnfo, Scopus y PubMed de la investigación sobre las variables de interés publicada desde el año 2000. Se analizaron un total de 35 estudios independientes pertenecientes a 33 artículos que cumplían los criterios de inclusión. La muestra total estaba compuesta por 13,233 participantes. Los resultados muestran una relación negativa de intensidad media entre la autoestima y la procrastinación en estudiantes, por lo que aquellos estudiantes que muestran una menor autoestima presentan una mayor procrastinación. Los análisis de meta-regresión mostraron que la edad media y el sexo no son variables moderadoras estadísticamente significativas; mientras que el análisis de subgrupos tampoco muestra que el nivel educativo modere el tamaño del efecto. No se aprecia la presencia de sesgo de publicación. Se comentan las implicaciones y las limitaciones del trabajo. Se señala la pertenencia de implementar programas para aumentar la autoestima de los estudiantes.

\section{Abstract}

Procrastination is a common behavior in academic field associated with various negative consequences. Self-esteem, understood as a global attitude towards oneself, is one of the variables that has been associated with academic procrastination. The objective of this work is to examine the relationship between self-esteem and procrastination in students using meta-analytical techniques. A bibliographic search was carried out in the Psyclnfo, Scopus and PubMed databases of the research on the variables of interest published since 2000. A total of 35 independent studies belonging to 33 articles that met the inclusion criteria were analyzed. The total sample consisted of 13,233 participants. The results show a negative relationship of medium intensity between self-esteem and procrastination in students, so that those students who show lower self-esteem have higher procrastination. The meta-regression analysis showed that mean age and sex are not statistically significant moderating variables; while the subgroup analysis does not show that the educational level moderates the effect size. The presence of publication bias is not appreciated. The implications and limitations of the work are discussed. The belonging of implementing programs to increase the self-esteem of the students is pointed out.

\section{Palabras clave / Keywords}

Educación, respeto de sí mismo, estudio bibliográfico, enseñanza secundaria, enseñanza superior, procrastinación, diferencia individual, personalidad.

Education, self-esteem, literature reviews, secondary education, higher education, procrastination, individual differences, personality. 


\section{Introducción}

En los últimos años, se han planteado diferentes definiciones en cuanto al concepto de la procrastinación. Si bien la mayoría de las mismas comparten la idea de que se trata de la dilación de diferentes tareas que estaban previstas, incluso cuando es conocido que por ello se actúa en contra de los intereses de uno mismo (Rebetez et al., 2016; Steel, 2011), este acuerdo no es tan claro en cuanto a las causas de este proceso y la relación que presenta con otros factores personales. Cada vez hay un mayor interés por este asunto, tal y como ponen de manifiesto los trabajos que se están realizando, dado que la procrastinación es una problemática muy extendida en la sociedad actual, en diversas culturas y en los diferentes ámbitos de la vida de la persona, considerándose como una conducta inadecuada y aversiva que dificulta la inserción efectiva de la persona en su cultura (Sirois y Pychyl, 2013). Si comprendemos los motivos por los que las personas procrastinan, podremos intervenir para reducir dicho comportamiento y mejorar, por tanto, la vida en sociedad. El paso inicial para diseñar y aplicar estrategias de intervención efectivas debe ser, por tanto, descubrir y profundizar en la comprensión de los aspectos que se asocian con la reducción de la procrastinación (Zhang et al., 2018).

La situación que acabamos de describir, se puede transferir al ámbito académico, concretándose en la demora voluntaria de ciertas tareas del ámbito educativo por parte de los estudiantes, aun conociendo que dicho retraso influirá negativamente en su rendimiento (Goroshit, 2018; Gustavson y Miyake, 2017; Steel y Klingsieck, 2016). Autores como Burgos-Torre y Salas-Blas (2020), han encontrado altos porcentajes de estudiantes que postergan al menos una tarea, llegando al 90\% en tareas puntuales (Sommer y Haug, 2012) y al $70 \%$ en la procrastinación de tareas de manera regular (Klingsieck et al., 2013). Si conocemos las causas concretas por las que los estudiantes tienden a procrastinar, podremos ayudarles a disminuir dicha conducta y, por tanto, a tener un rendimiento más óptimo.

En los estudios sobre procrastinación académica que se han mencionado no se encontraron diferencias por nacionalidad ni por sexo; tampoco Zhang et al. (2018) encontraron discrepancias significativas en relación al sexo, especialidad, grado y ciudad de origen en su análisis de la prevalencia de la procrastinación y su relación con la autoestima en el ámbito universitario. Sin embargo, otros trabajos, como los realizados por Limone et al. (2020), encontraron que las mujeres presentaban menores puntuaciones que los hombres en relación a la procrastinación y a la administración del tiempo. Por tanto, parece que los estudiantes masculinos tienden en mayor medida que sus compañeras a dilatar la realización de las tareas.

La procrastinación en el ámbito educativo se ha relacionado con diversas consecuencias negativas como bajo rendimiento académico (Hidalgo-Fuentes et al., 2021), mayor número de comportamientos académicos deshonestos (Clariana et al., 2012), disminución del compromiso académico (Aspée et al., 2021) o mayor probabilidad de abandonar los estudios (Bäulke et al., 2018).

La autoestima es una de las variables que se ha relacionado con la procrastinación académica, entendiendo la misma como una actitud global que presenta la persona hacía sí misma (Leary y MacDonald, 2003). Altos grados de autoestima se han vinculado a una mayor permanencia en el sistema educativo, un mayor bienestar subjetivo emocional y un mejor desempeño futuro profesional (San Martín y Barra, 2013). En contraposición, un bajo nivel de autoestima puede relacionarse con dificultades de adaptación social o, incluso, con una mayor probabilidad de comportamientos suicidas (Montes y Tomás, 2016). De estos hallazgos se deriva la importancia y necesidad de analizar la autoestima de los estudiantes para poder intervenir de cara a mejorar los niveles de dicha variable, mejorando así no solo su rendimiento a nivel académico, sino también su satisfacción y felicidad integral.

Respecto al estudio de la relación entre la procrastinación y la autoestima, son escasos los trabajos existentes. Pese a ello, sí se ha analizado la existencia de una asociación entre la procrastinación y la impulsividad (Gustavson et al., 2014) y, en consecuencia, su asociación con una falta de autorregulación a la hora de actuar en diferentes ámbitos (Steel y Klingsieck, 2016).

Desde la perspectiva de la regulación emocional, se apoya la idea de que las personas procrastinan para evitar realizar conductas que les provocan emociones negativas (Sioris y Pychyl, 2016; Zhang y Feng, 2019). Sin embargo, el propio hecho de retrasar dichas conductas puede conllevar un aumento de la ansiedad (Rozental et al., 2018) y, por ende, un efecto negativo en nuestra autoestima. Adicionalmente, diversos estudios han puesto de manifiesto que la procrastinación tiene una influencia negativa en la salud psicológica, pudiendo reducir el bienestar subjetivo (Balkis y Duru, 2015) o, incluso, potenciando la depresión (Flett et al, 2016; Kınık y Odacı, 2020), altos niveles de ansiedad (Yang et al., 2019), o estrés (Sioris y Giguère, 2018). Más concretamente, en el ámbito académico, se ha encontrado que la procrastinación afecta el rendimiento académico, lo cual promueve una falta de satisfacción con la vida académica, asociada a una baja autoestima 
(Hajloo, 2014; Hen y Goroshit, 2014). Según Zhang et al. (2018), aquellos estudiantes que poseen un nivel bajo de autoestima, pueden presentar un mayor grado de procrastinación debido, al menos en parte, a su percepción de baja autoeficacia ante las tareas académicas. En línea con estos autores, Abdi-Zarrin et al. (2020), también encontraron que el miedo al fracaso se relaciona con una mayor procrastinación o demora de las tareas académicas y, por tanto, también con una baja autoestima.

A raíz de lo anteriormente expuesto, el objetivo del presente trabajo es sintentizar los resultados de los estudios que desde el año 2000 han evaluado la relación entre la autoestima y la procrastinación en estudiantes abordando las siguientes preguntas de investigación: 1) ¿cuál es la intensidad y el signo de la relación entre la autoestima y la procrastinación en el ámbito académico? y 2) ¿está la asociación entre la autoestima y la procrastinación moderada por el sexo, la edad, el nivel educativo de los estudiantes y el área geográfica en la que se realizaron los estudios?

\section{Metodología}

\subsection{Búsqueda sistemática y selección de estudios}

Con el objetivo de localizar estudios que presentasen datos sobre la relación entre autoestima y procrastinación en el ámbito académico se llevó a cabo una búsqueda durante los meses de octubre y noviembre de 2021 en las bases de datos Psyclnfo, Scopus y Pubmed. Se utilizaron las palabras clave "procrastination", "self-esteem", "self-worth", "self-liking" y "self-concept" en los campos título, resumen y palabras clave, combinándolas mediante los operadores boleanos "and" y "or". La búsqueda estuvo restringida a artículos revisados por pares y limitada temporalmente a artículos publicados a partir del año 2000. Con la finalidad de identificar estudios relevantes adicionales, se realizaron dos búsquedas de forma manual: una de los trabajos que citaban a los artículos seleccionados (búsqueda hacia delante) y otra sobre todos los trabajos referenciados en los artículos seleccionados (búsqueda hacia atrás).

Los criterios de inclusión establecidos para incluir los artículos recuperados en los análisis meta-analíticos fueron los siguientes: 1) estudios cuantitativos transversales publicados en revistas científicas revisadas por pares a partir del año 2000 , 2) publicados en español o inglés, 3) presentan al menos una medida de autoestima o autoconcepto y una medida de procrastinación, 4) los sujetos de la muestra son estudiantes, 5) presentan un coeficiente de correlación de Pearson ( $r$ ) entre las medidas de autoestima y procrastinación o información estadística suficiente para estimarlo, 6) informan del tamaño muestral y 7) el texto completo es accesible. Así mismo, se tuvieron en cuenta los siguientes criterios de exclusión: 1) artículos teóricos, revisiones sistemáticas o meta-análisis y artículos longitudinales, 2) libros, capítulos de libros, actas de congreso u otros tipos de publicaciones no revisadas por pares, 3) acceso restringido a la publicación, 4) estudios sin datos estadísticos suficientes para calcular la correlación de Pearson entre la autoestima y la procrastinación, 5) los participantes no son estudiantes y 6) estudios duplicados (en el caso de solapamiento de parte de la muestra se ha optado por incluir aquellos estudios con un mayor tamaño muestral).

La Figura 1 presenta un resumen del proceso de búsqueda y selección de estudios. La búsqueda inicial dio como resultado 283 artículos recuperados. En un primer paso, se eliminaron 100 artículos duplicados. Tras el examen del título y el resumen de los artículos restantes, se eliminaron 122 artículos adicionales debido a su irrelevancia respecto al objetivo del meta-análisis. El texto completo de los 62 artículos restantes fue revisado para valorar el cumplimiento de los criterios de inclusión, dando como resultado 31 artículos seleccionados con 33 estudios independientes. Tras las búsquedas manuales hacia delante y hacia atrás se añadieron dos estudios adicionales. 


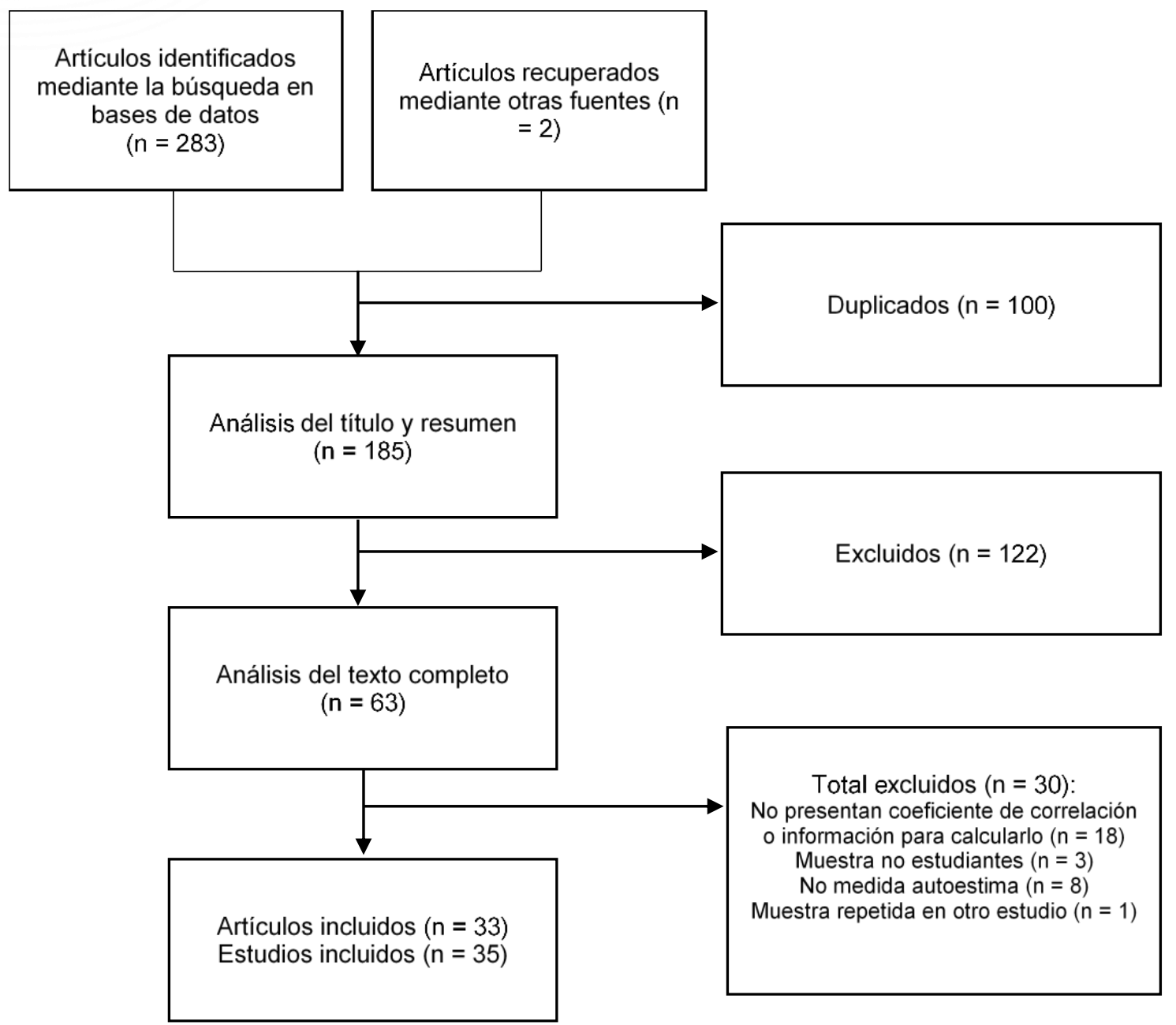

Figura 1. Diagrama de flujo del proceso de búsqueda y selección de estudios

\subsection{Codificación de estudios}

Las características extraídas de cada estudio fueron las siguientes: autor/es, año de publicación, país, área geográfica (codificada como Occidente, Oriente Medio o Asia) tamaño muestral, edad media de la muestra, sexo (codificado como porcentaje de mujeres en la muestra), nivel educativo de los participantes (secundaria o universitario), medida/s de autoestima, medida/s de procrastinación y coeficiente de correlación de Pearson entre autoestima y procrastinación. En caso de datos faltantes, se envió un correo electrónico al autor de contacto solicitando los mismo, aquellos que no respondieron a la solicitud o carecían de los datos figuran como no disponibles. Los estudios fueron codificados de manera independiente por dos de los autores, resolviéndose las discrepancias mediante consenso. En los estudios que no utilizaban la correlación de Pearson ( $r$ ) como tamaño del efecto, se procedió a su conversión mediante la fórmula adecuada. Específicamente, en el estudio de Pychyl et al. (2002) se convirtió el coeficiente $\beta$ en $r$ mediante la fórmula $r$ $=\beta+.05 \lambda$ (siendo $\lambda=0$ cuando $\beta$ es negativa y $\lambda=1$ cuando $\beta$ es positiva); mientras que en el estudio de Uribe et al. (2020) se transformó el coeficiente de correlación de Spearman en $r$ a través de la fórmula $r=$ $2^{*} \operatorname{sen}\left(\mathrm{rho}^{*}(\pi / 6)\right)$. En los estudios que presentaban varias correlaciones entre la autoestima y la procrastinación al haber utilizados varios instrumentos para medir la misma variable, se agregaron todas las correlaciones utilizando la fórmula para combinar correlaciones propuesta por Hunter y Schmidt (2004). 


\subsection{Análisis de datos}

Los análisis se llevaron a cabo aplicando un modelo de efectos aleatorios con un intervalo de confianza (IC) al 95\% mediante el paquete Major para Jamovi (Hamilton, 2018). Se utilizó asimismo el programa MetaEssentials 1.5 (Suurmond et al, 2017) para la realización del análisis de subgrupos.

Antes de proceder a los análisis, y con la finalidad de aproximar las correlaciones de Pearson a una distribución normal (Cohn y Becker, 2003), dichas correlaciones fueron transformadas en puntuaciones $Z$ de Fisher mediante la fórmula $Z=0.5^{*} \ln [(1+r) /(1-r)]$. El tamaño del efecto global y su IC fueron transformados de vuelta a correlaciones de Pearson para una mejor interpretación siguiendo la recomendación de Borenstein et al. (2021).

La heterogeneidad fue evaluada mediante la prueba $Q$ de Cochran y el estadístico $R$ (Higgins y Thompson, 2002). Para la valoración del riesgo de sesgo de publicación, y siguiendo la recomendación Botella y SánchezMeca (2015) de utilizar diversos métodos, se emplearon el test de regresión de Egger, el cálculo del número crítico de seguridad con el método de Rosenthal y la prueba de correlación de rangos de Begg y Mazumdar. Para finalizar, y con el objetivo de examinar los posibles efectos de moderación de las variables sexo, edad media, nivel educativo de los participantes y área geográfica en la que se condujeron los estudios, se realizaron análisis de meta-regresión para las dos primeras, al estar codificadas como variables continuas; y sendos análisis de subgrupos para las dos últimas, al ser variables cualitativas.

\section{Resultados}

La base final utilizada para los análisis meta-analíticos estuvo formada por 35 estudios independientes pertenecientes a 33 artículos, con un total de 13,233 sujetos (véase la Tabla 1). El tamaño muestral de los estudios varía entre los 80 y los 1424 participantes. La edad media oscila entre los 13.65 y los 23.33 años, con una edad media global de 20.09 años. En 32 de los estudios hay un mayor porcentaje de mujeres en la muestra, mientras que solo tres presentan un mayor porcentaje de participantes masculinos. El país con una mayor representación en los análisis fue Turquía con diez estudios, seguido de China y EEUU con cinco estudios cada uno.

Tabla 1

Variables sociodemográficas de los estudios incluidos

\begin{tabular}{|c|c|c|c|c|c|c|}
\hline Estudio & País & $\begin{array}{c}\text { Área } \\
\text { geográfica }\end{array}$ & Tamaño muestral & Edad media & $\begin{array}{c}\text { Nivel } \\
\text { educativo }\end{array}$ & Sexo (\% mujeres) \\
\hline Pychyl et al., 2002 & Canadá & Occidente & 105 & 13,65 & Universitario & 57,14 \\
\hline Batool et al., 2017 & Pakistán & $\begin{array}{l}\text { Oriente } \\
\text { Medio }\end{array}$ & 502 & 22,64 & Universitario & 58,17 \\
\hline Chen, 2017 & China & Asia & 384 & 15,13 & Secundaria & 50,4 \\
\hline Zhang et al., 2018 & China & Asia & 1184 & nd & Universitario & 68,7 \\
\hline Klibert et al., 2011 (1) & EEUU & Occidente & 336 & 20,69 & Universitario & 100 \\
\hline Klibert et al., 2011 (2) & EEUU & Occidente & 139 & 21,37 & Universitario & 0 \\
\hline Beck et al., 2000 & EEUU & Occidente & 169 & 19,41 & Universitario & 62,13 \\
\hline Klassen et al., 2008 & Canadá & Occidente & 261 & 23,33 & Universitario & 81 \\
\hline Tan et al., 2015 & China & Asia & 304 & 21,6 & Universitario & 50,3 \\
\hline Wang y Lei, 2021 & China & Asia & 762 & 16,79 & Secundaria & 56 \\
\hline Selçuk et al., 2021 & Turquía & $\begin{array}{l}\text { Oriente } \\
\text { Medio }\end{array}$ & 353 & 16,86 & Secundaria & 48,44 \\
\hline Kınık y Odacı, 2020 & Turquía & $\begin{array}{l}\text { Oriente } \\
\text { Medio }\end{array}$ & 862 & 20,88 & Universitario & 71,3 \\
\hline Barutçu-Yıldırım y Demir, 2020 & Turquía & $\begin{array}{l}\text { Oriente } \\
\text { Medio }\end{array}$ & 801 & nd & Universitario & 50,4 \\
\hline Batool, 2020 & Australia & Occidente & 502 & 22,64 & Universitario & 58,17 \\
\hline Boysan y Kiral, 2017 & Turquía & $\begin{array}{l}\text { Oriente } \\
\text { Medio }\end{array}$ & 242 & 20,98 & Universitario & 66,94 \\
\hline Chen et al., 2016 & China & Asia & 199 & 19,3 & Universitario & 80,9 \\
\hline
\end{tabular}




\begin{tabular}{|c|c|c|c|c|c|c|}
\hline Estudio & País & $\begin{array}{c}\text { Área } \\
\text { geográfica }\end{array}$ & Tamaño muestral & Edad media & $\begin{array}{c}\text { Nivel } \\
\text { educativo }\end{array}$ & Sexo (\% mujeres) \\
\hline Clarke y MacCann, 2016 & Australia & Occidente & 484 & 19,7 & Universitario & 64,9 \\
\hline Athulya et al., 2016 & India & Asia & 192 & 21,22 & Universitario & 68,8 \\
\hline Rebetez et al., 2015 & Suiza & Occidente & 180 & 21,85 & Universitario & 85 \\
\hline Özer et al., 2013 & Turquía & $\begin{array}{l}\text { Oriente } \\
\text { Medio }\end{array}$ & 622 & 21,3 & Universitario & 50,32 \\
\hline Macher et al., 2012 & Austria & Occidente & 147 & 20,8 & Universitario & 76,19 \\
\hline Klassen y Kuzucu, 2009 (1) & Turquía & $\begin{array}{l}\text { Oriente } \\
\text { Medio }\end{array}$ & 248 & nd & Secundaria & 100 \\
\hline Klassen y Kuzucu, 2009 (2) & Turquía & $\begin{array}{l}\text { Oriente } \\
\text { Medio }\end{array}$ & 260 & nd & Secundaria & 0 \\
\hline Di Fabio, 2006 & Italia & Occidente & 258 & 17,81 & Secundaria & 56,98 \\
\hline Harrington, 2005 & Reino Unido & Occidente & 86 & nd & Universitario & 78 \\
\hline Sirois, 2004 & Canadá & Occidente & 80 & 19,5 & Universitario & 71,25 \\
\hline Fee y Tangney, 2000 & EEUU & Occidente & 86 & 21,86 & Universitario & 60 \\
\hline Ferrari, 2000 & EEUU & Occidente & 142 & 21,1 & Universitario & 56,34 \\
\hline Niu, 2021 & Tibet & Asia & 559 & 18 & Secundaria & 63,5 \\
\hline Uribe et al., 2020 & Perú & Occidente & 1424 & 18,26 & Universitario & 55,76 \\
\hline Brando-Garrido et al., 2020 & España & Occidente & 237 & 22,81 & Universitario & 85,23 \\
\hline Hajloo, 2014 & Irán & $\begin{array}{l}\text { Oriente } \\
\text { Medio }\end{array}$ & 140 & 20,5 & Universitario & 62,86 \\
\hline Duru y Balkis, 2014 & Turquía & $\begin{array}{l}\text { Oriente } \\
\text { Medio }\end{array}$ & 261 & 21,34 & Universitario & 77,78 \\
\hline Duru y Balkis, 2017 & Turquía & $\begin{array}{l}\text { Oriente } \\
\text { Medio }\end{array}$ & 348 & 21,3 & Universitario & 73 \\
\hline Kandemir et al., 2014 & Turquía & $\begin{array}{l}\text { Oriente } \\
\text { Medio }\end{array}$ & 374 & nd & Universitario & 70,86 \\
\hline
\end{tabular}

En la Tabla 2 se observan los instrumentos utilizados para evaluar la autoestima y la procrastinación en los diferentes estudios incluidos. La prueba más utilizada para medir la procrastinación fue la General Procrastination Scale (Lay, 1986), mientras que en el caso de la autoestima la mayoría de estudios emplearon la Rosenberg Self-esteem Scale (Rosenberg, 1965).

\section{Tabla 2}

Instrumentos utilizados en los estudios incluidos para medir autoestima y procrastinación

\begin{tabular}{|c|c|c|}
\hline Estudio & Instrumento autoestima & Instrumento procrastinación \\
\hline Pychyl et al., 2002 & $\begin{array}{l}\text { Self-Perception Profile for } \\
\text { Adolescents }\end{array}$ & General Procrastination Scale \\
\hline Batool et al., 2017 & Rosenberg Self-esteem Scale & $\begin{array}{l}\text { Procrastination Assessment Scale for } \\
\text { Students }\end{array}$ \\
\hline Chen, 2017 & $\begin{array}{l}\text { Self-Perception Profile for } \\
\text { Adolescents }\end{array}$ & General Procrastination Scale \\
\hline Zhang et al., 2018 & Rosenberg Self-esteem Scale & $\begin{array}{l}\text { Procrastination Assessment Scale for } \\
\text { Students }\end{array}$ \\
\hline Klibert et al., 2011 (1) & Rosenberg Self-esteem Scale & Tuckman's Procrastination Scale \\
\hline Klibert et al., 2011 (2) & Rosenberg Self-esteem Scale & Tuckman's Procrastination Scale \\
\hline Beck et al., 2000 & Rosenberg Self-esteem Scale & $\begin{array}{l}\text { Procrastination Assessment Scale for } \\
\text { Students }\end{array}$ \\
\hline Klassen et al., 2008 & Rosenberg Self-esteem Scale & Tuckman's Procrastination Scale \\
\hline Tan et al., 2015 & Rosenberg Self-esteem Scale & Aitken Procrastination Inventory \\
\hline Wang y Lei, 2021 & Rosenberg Self-esteem Scale & General Procrastination Scale \\
\hline Selçuk et al., 2021 & $\begin{array}{l}\text { Academic Self-Concept Scale } \\
\text { (modificada) }\end{array}$ & $\begin{array}{l}\text { General Procrastination Scale } \\
\text { (modificada) }\end{array}$ \\
\hline
\end{tabular}




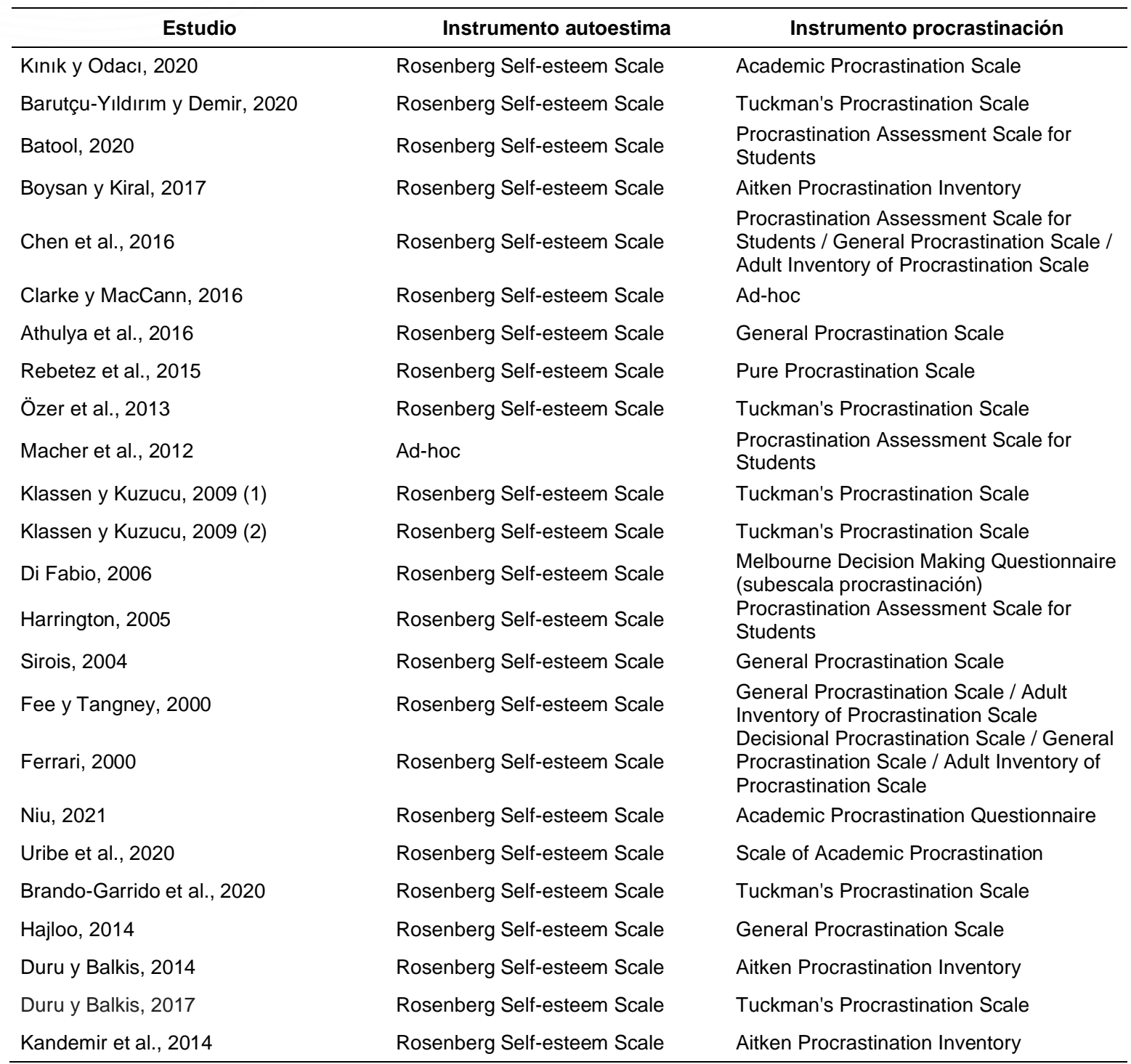

Los análisis realizados muestran una asociación negativa estadísticamente significativa entre la autoestima y la procrastinación en estudiantes, con un tamaño del efecto estimado de $Z r=-0.32$ (IC 95\% [-0.36, -0.27]; $p$ $<.001$ ), lo que convertido en correlaciones de Pearson resulta en $r=-.31$ (IC 95\% [-.35, -.26]), por lo que según el criterio propuesto por Cohen (1988) puede interpretarse como una asociación de intensidad media. La Figura 2 se presenta el forest plot con el tamaño del efecto y el IC de los estudios incluidos en el metaanálisis. 


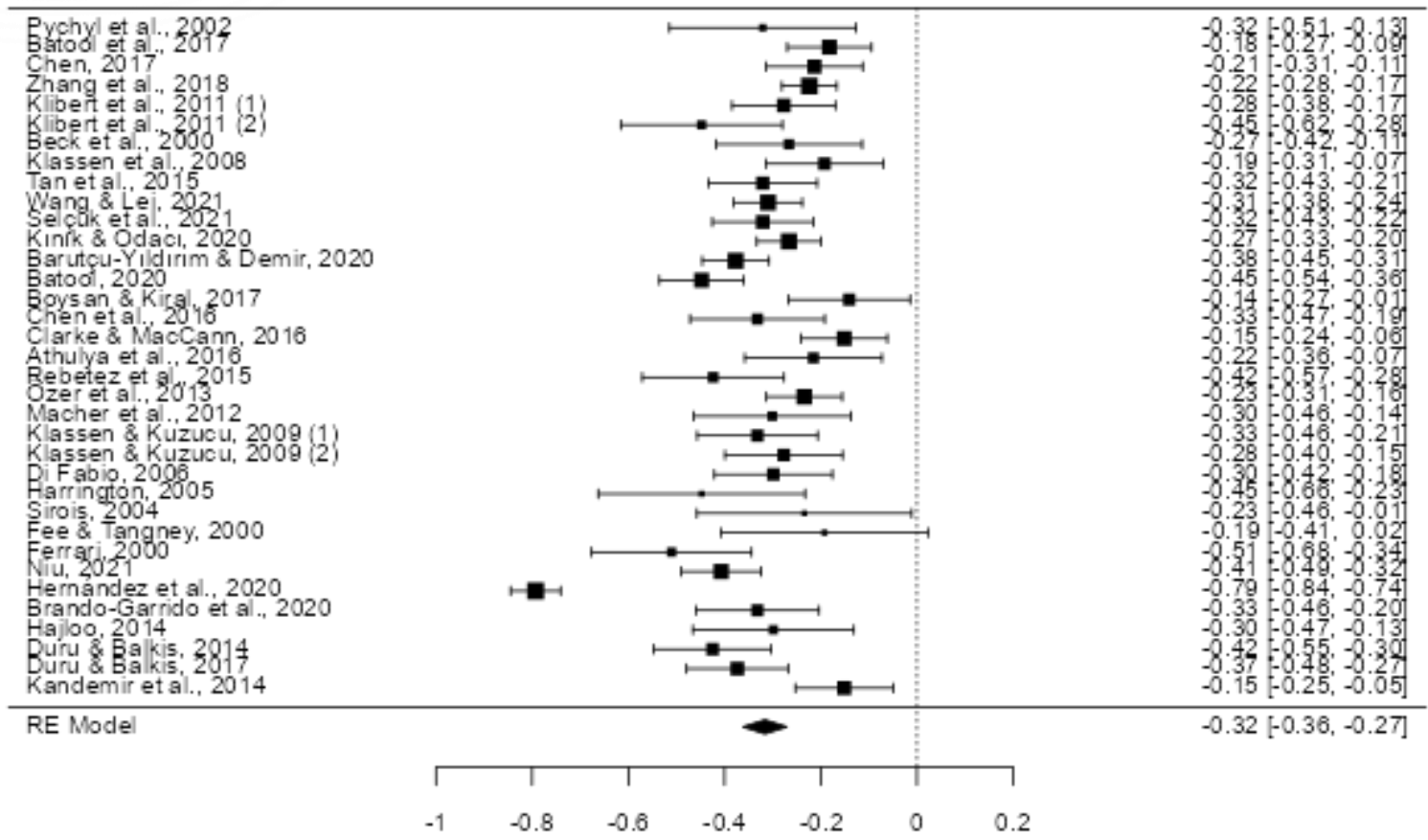

Figura 2. Forest plot

La prueba $Q$ de Cochran arrojó un resultado de $Q=412.985$ ( $p<.001)$, por ello la hipótesis de homogeneidad es rechazada; mientras que el índice $R$, que evalúa el porcentaje real de heterogeneidad de los estudios incluidos en el meta-análisis, presentó un resultado de $84.59 \%$, considerado un porcentaje de heterogeneidad alto según el criterio sugerido por Higgins et al., (2003), lo que podría estar indicando que el tamaño del efecto está moderado por otras variables.

En cuanto al riesgo de sesgo de publicación, tanto el test de regresión de Egger ( $p=.631)$ como la prueba de correlación de rangos de Begg y Mazumdar $(p<.201)$ presentan valores no significativos, por ello se descarta la presencia de sesgo de publicación. Asimismo, el cálculo del número crítico de seguridad según el método de Rosenthal confirma la ausencia de sesgo de publicación al presentar un resultado de $n=15,599(p<.001)$, por lo tanto harían falta 15,599 estudios no publicados con un tamaño del efecto de cero para convertir el valor $p$ en no significativo; este número de estudios supera considerablemente el valor crítico para este metaanálisis de 185 estudios, calculado mediante la fórmula $\left(5^{\star} \mathrm{k}\right)+10$, donde $\mathrm{k}$ el número de estudios incluidos en el meta-análisis (Botella y Sánchez-Meca, 2015).

Los análisis de meta-regresión en relación a las posibles variables cuantitativas moderadoras mostraron que ni la edad media $(p=.638)$ ni el sexo de los estudiantes $(p=.569)$ moderan la relación entre la autoestima y la procrastinación (véase Tabla 3). El análisis de subgrupos para valorar el posible efecto moderador del nivel educativo (secundaria vs. universidad) mostró un resultado de $Q_{\text {between }}(g \mid=1)=0.01, p=.916$, por consiguiente, esta variable tampoco modera significativamente el tamaño del efecto entre autoestima y procrastinación. Así mismo, el análisis de subgrupos para examinar el efecto moderador del área geográfica en la que se condujeron los estudios (Occidente vs. Oriente Medio vs. Asia) presentó un resultado de $Q$ between $(\mathrm{gl}=2)=1.09, \mathrm{p}=.581$, no resultando tampoco una variable moderadora de la asociación entre la autoestima y las variables estudiadas. 
Tabla 3

Análisis de variables moderadoras

\begin{tabular}{lcccc}
\hline $\begin{array}{l}\text { Moderadores } \\
\text { continuos }\end{array}$ & Coeficiente & Error estándar & $\mathbf{Z}$ & $\boldsymbol{p}$ \\
\hline Edad media & .006 & .012 & 0.47 & .638 \\
Sexo & .000 & .001 & 0.57 & .569 \\
\hline $\begin{array}{l}\text { Moderadores } \\
\text { categóricos }\end{array}$ & $\mathbf{r}$ & IC 95\% & Qbetween & $\boldsymbol{p}$ \\
\hline $\begin{array}{l}\text { Nivel educativo } \\
\text { Secundaria }\end{array}$ & -.31 & $-.37,-.26$ & & \\
$\begin{array}{l}\text { Universitario } \\
\text { Área geográfica }\end{array}$ & -.32 & $-.37,-.26$ & & .016 \\
$\begin{array}{l}\text { Occidente } \\
\text { Oriente Medio }\end{array}$ & -.35 & $-.44,-.27$ & & \\
Asia & -.28 & $-.34,-.22$ & & .09 \\
\hline
\end{tabular}

\section{Discusión}

Después de una búsqueda sistemática de artículos relacionados con el tema, se recuperaron 35 estudios independientes, perteneciente a 33 artículos, con una muestra combinada de 13,233 estudiantes sobre los que se llevaron a cabo los análisis meta-analíticos.

Todos los estudios analizados presentaban correlaciones negativas entre la autoestima y la procrastinación; mientras que el resultado global del meta-análisis mostró una relación estadísticamente significativa negativa de intensidad media entre ambas variables, en consecuencia, los estudiantes que presentaban una menor autoestima, mostraban a su vez niveles más elevados de procrastinación académica. Este resultado goza de fiabilidad y robustez, ya que se descartó la existencia de sesgo de publicación mediante tres métodos diferentes. La procrastinación ha sido conceptualizada como un mecanismo de protección frente a una autoestima baja o vulnerable (Steel, 2007), lo que es congruente con los resultados encontrados. Asimismo, la procrastinación en el ambiente educativo se ha relacionado con el miedo al fracaso y una baja satisfacción académica, que también son más comunes en estudiantes con una autoestima baja (Hen y Goroshit, 2014; Zhang et al., 2018).

En relación con las posibles variables moderadoras del tamaño del efecto, ninguna de las analizadas resultó ser un moderador estadísticamente significativo. En cuanto a la edad, la mayoría de los estudios incluidos en el meta-análisis presentan valores similares para la edad media de los participantes al estar centrados en estudiantes, por lo que la falta de heterogeneidad en esta variable podría ser la causa de dicho resultado. En relación al sexo, aunque hay evidencia de que los estudiantes varones son más procrastinadores que las mujeres (Balkis y Erdinç, 2017; Dominguez-Lara et al., 2019; Limone et al., 2020), hay discrepancias sobre diferencias en la autoestima en relación a esta variable, encontrando algunos estudios una mayor autoestima en varones (Bleidorn et al., 2016; Povedano et al., 2011), mientras que otros no han hallado diferencias significativas en función del sexo (Kong et al., 2015). Esta divergencia podría provocar que el sexo no se haya mostrado como moderador de la relación entre la autoestima y la procrastinación. De manera similar, el área geográfica en la que se realizaron los estudios no modera la asociación entre las variables objeto de interés en el presente meta-análisis, al no presentar diferencias significativas el tamaño del efecto encontrado en los tres subgrupos analizados. Por último, y a pesar de que la procrastinación suele ser más frecuente en alumnos universitarios que de secundaria, esta variable tampoco ha resultado ser un moderador de la asociación entre la autoestima y la procrastinación.

A pesar de la fiabilidad de los resultados encontrados, hay que tener presente ciertas limitaciones a la hora de interpretarlos. En primer lugar, al haberse centrado en estudios transversales, no se pueden establecer relaciones causales entre la autoestima y la procrastinación, por lo que futuros estudios de carácter longitudinal podrían ayudar a esclarecer la direccionalidad de la relación entre ambas variables, así como su variación a lo largo de la vida académica de los estudiantes. Una segunda limitación es que debido a que solo se han incluido en los análisis estudios publicados en inglés o español, cabe la posibilidad de haber perdidos estudios escritos en otros idiomas. En tercer lugar, aunque nuestro trabajo abarca un periodo temporal muy amplio al haber recogido estudios publicados desde el año 2000, los resultados encontrados podrían ser diferentes si hubiéramos incluido estudios anteriores. Una cuarta limitación podría ser una 
sobrerrepresentación de estudiantes femeninas en las muestras de los estudios analizados, ya que la mayoría de ellos presentan un mayor porcentaje de mujeres entre sus participantes, pero dado que el sexo no se ha mostrado como un moderador del tamaño del efecto no parece que los resultados se hayan visto afectados por ello. Por último, no se han recuperado estudios con alumnos del nivel de educación primaria, por lo que, de cara al futuro, sería recomendable realizar estudios sobre la autoestima y la procrastinación en este nivel educativo, ya que los niños en el rango de edad correspondiente a este nivel muestran patrones de autoestima diferenciados de los encontrados posteriormente en los adolescentes (Robins y Tzesniewski, 2005).

\section{Conclusiones}

La procrastinación académica tiene importantes consecuencias negativas tanto dentro del ámbito educativo como en el bienestar general de los estudiantes. El presente meta-análisis ha mostrado evidencias de una asociación estadísticamente significativa de signo negativo y de intensidad media entre la autoestima y la procrastinación en el ámbito académico, sin que dicha relación esté moderada por la edad, el sexo, el nivel educativo y el área geográfica, variables incluidas en el estudio. La procrastinación es un comportamiento muy frecuente en el ámbito académico que conlleva consecuencias negativas para los estudiantes como bajo rendimiento académico (Hidalgo-Fuentes et al., 2021) aumento de comportamiento académicos deshonestos (Aspée et al., 2021) o mayor probabilidad de abandono de los estudios (Clariana en al., 2012). En relación a las implicaciones prácticas de este trabajo, la asociación negativa encontrada entre la autoestima y la procrastinación en la esfera educativa apunta a la pertinencia de establecer programas dirigidos a aumentar la autoestima entre los estudiantes que podrían ayudar a reducir la procrastinación, habiendo demostrado sendos meta-análisis que las intervenciones centradas en la mejora de la autoestima se han probado eficaces tanto en niños y adolescentes (Haney \& Durlak, 1998) como en adultos (Niveau et al., 2021). Como se ha comentado anteriormente, investigaciones futuras deberían continuar examinando la relación entre la autoestima y la procrastinación en diferentes niveles educativos a los ya estudiados como la educación primaria o la formación profesional, así como tratar de establecer que variables tanto personales como metodológicas podrían estar moderando la intensidad de dicha relación.

\section{Referencias}

Las referencias con $\left(^{*}\right)$ están incluidas en el meta-análisis.

Abdi-Zarrin, S., Gracia, E., y Paixão, M. P. (2020). Prediction of academic procrastination by fear of failure and selfregulation. Educational Sciences: Theory and Practice, 20(3), 34-43. https://doi.org/10.12738/jestp.2020.3.003

*Athulya, J., Sudhir, P. M., y Philip, M. (2016). Procrastination, perfectionism, coping and their relation to distress and self-esteem in college students. Journal of the Indian Academy of Applied Psychology, 42(1), 82-91. Retrieved from https://bit.ly/3HSlih5

Balkis, M., y Duru, E. (2015). Procrastination, self-regulation failure, academic life satisfaction, and affective well-being: underregulation or misregulation form. European Journal of Psychology of Education, 31(3), 439-459. https://doi.org/10.1007/s10212-015-0266-5

Balkis, M., y Erdinç, D. U. R. U. (2017). Gender differences in the relationship between academic procrastination, satifaction with academic life and academic performance. Electronic Journal of Research in Educational Psychology, 15(1), 105-125. https://doi.org/10.14204/eirep.41.16042

*Barutçu-Yıldırım, F., y Demir, A. (2020). Self-handicapping among university students: The role of procrastination, test anxiety, self-esteem, and self-compassion. Psychological Reports, 123(3), 825-843. https://doi.org/10.1177/0033294118825099

*Batool, S. S. (2020). Academic achievement: Interplay of positive parenting, self-esteem, and academic procrastination. Australian Journal of Psychology, 72(2), 174-187. https://doi.org/10.1111/ajpy.12280

*Batool, S. S., Khursheed, S., y Jahangir, H. (2017). Academic procrastination as a product of low self-esteem: A mediational role of academic self-efficacy. Pakistan Journal of Psychological Research, 32(1), 195-211. Retrieved from https://bit.ly/3r6VN1G

Bäulke, L., Eckerlein, N., y Dresel, M. (2018). Interrelations between motivational regulation, procrastination and college dropout intentions. Unterrichtswissenschaft, 46(4), 461-479. https://doi.org/10.1007/s42010-018-0029-5

${ }^{*}$ Beck, B. L., Koons, S. R., y Milgrim, D. L. (2000). Correlates and consequences of behavioral procrastination: The effects of academic procrastination, self-consciousness, self-esteem and self-handicapping. Journal of Social Behavior and Personality, 15(5), 3-13. Retrieved from https://bit.ly/3rgcEz0

Bleidorn, W., Arslan, R. C., Denissen, J. J., Rentfrow, P. J., Gebauer, J. E., Potter, J., y Gosling, S. D. (2016). Age and gender differences in self-esteem-A cross-cultural window. Journal of personality and social psychology, 111(3), 396. https://doi.org/10.1037/pspp0000078

Borenstein, M., Hedges, L. V., Higgins, J. P., y Rothstein, H. R. (2021). Introduction to meta-analysis. John Wiley y Sons 
Botella, J. y Sánchez-Meca, J. (2015). Meta-Análisis en Ciencias Sociales y de la Salud. Editorial Síntesis

*Boysan, M., y Kiral, E. (2017). Associations between procrastination, personality, perfectionism, self-esteem and locus of control. British Journal of Guidance \& Counselling, 45(3), 284-296. https://doi.org/10.1080/03069885.2016.1213374

*Brando-Garrido, C., Montes-Hidalgo, J., Limonero, J. T., Gómez-Romero, M. J., y Tomás-Sábado, J. (2020). Relationship of academic procrastination with perceived competence, coping, self-esteem and self-efficacy in nursing students. Enfermería Clínica (English Edition), 30(6), 398-403. https://doi.org/10.1016/j.enfcle.2019.07.013

Burgos-Torre, K. S., y Salas-Blas, E. (2020). Procrastinación y Autoeficacia académica en estudiantes universitarios limeños. Propósitos y Representaciones, 8(3). e790. https://doi.org/10.20511/pyr2020.v8n3.790

${ }^{*}$ Chen, B. B. (2017). Parent-adolescent attachment and procrastination: The mediating role of self-worth. The Journal of Genetic Psychology, 178(4), 238-245. https://doi.org/10.1080/00221325.2017.1342593

${ }^{*}$ Chen, B. B., Shi, Z., y Wang, Y. (2016). Do peers matter? Resistance to peer influence as a mediator between selfesteem and procrastination among undergraduates. Frontiers in Psychology, 7, 1529. https://doi.org/10.3389/fpsyg.2016.01529

Clariana, M., Gotzens, C., del Mar Badia, M., y Cladellas, R. (2012). Procrastination and cheating from secondary school to university. Electronic Journal of Research in Educational Psychology, 10(2), 737-754. https://doi.org/10.25115/eirep.v10i27.1525

${ }^{*}$ Clarke, I. E., y MacCann, C. (2016). Internal and external aspects of self-handicapping reflect the distinction between motivations and behaviours: Evidence from the Self-handicapping Scale. Personality and Individual Differences, 100, 6-11. https://doi.org/10.1016/j.paid.2016.03.080

Cohen, J. (1988). Statistical Power Analysis for the Behavioral Sciences. Lawrence Erlbaum Associates.

Cohn, L. D., y Becker, B. J. (2003). How meta-analysis increases statistical power. Psychological methods, 8(3), 243. Retrieved from https://bit.ly/3r7F8uN

*Di Fabio, A. (2006). Decisional procrastination correlates: personality traits, self-esteem or perception of cognitive failure?. International Journal for Educational and Vocational Guidance, 6(2), 109-122. https://doi.org/10.1007/s10775-006-9000-9

Dominguez-Lara, S., Prada-Chapoñan, R., y Moreta-Herrera, R. (2019). Gender differences in the influence of personality on academic procrastination in Peruvian college students. Acta Colombiana de Psicología, 22(2), 125147. https://doi.org/10.14718/ACP.2019.22.2.7

*Duru, E., y Balkis, M. (2014). The roles of academic procrastination tendency on the relationships among self doubt, self esteem and academic achievement. Education and Science, 39(173), 274-289. Retrieved from https://bit.ly/313cGzD

*Duru, E., y Balkis, M. (2017). Procrastination, self-esteem, academic performance, and well-being: A moderated mediation model. International Journal of Educational Psychology, 6(2), 97. https://doi.org/10.17583/ijep.2017.2584

${ }^{*}$ Fee, R. L., y Tangney, J. P. (2000). Procrastination: A means of avoiding shame or guilt?. Journal of Social Behavior and Personality, 15(5), 167-184. Retrieved from https://bit.ly/2ZkiKTy

${ }^{*}$ Ferrari, J. R. (2000). Procrastination and attention: Factor analysis of attention deficit, boredomness, intelligence, selfesteem, and task delay frequencies. Journal of Social Behavior and Personality, 15(5), 185-196. Retrieved from https://bit.ly/3HVPvlq

Flett, A. L., Haghbin, M., y Pychyl, T. A. (2016). Procrastination and Depression from a Cognitive Perspective: An Exploration of the Associations Among Procrastinatory Automatic Thoughts, Rumination, and Mindfulness. Journal of Rational-Emotive and Cognitive-Behavior Therapy, 34(3), 169-186. https://doi.org/10.1007/s10942-016-0235-1

Goroshit, M. (2018). Academic procrastination and academic performance: An initial basis for intervention. Journal of Prevention \& Intervention in the Community, 46(2), 131-142. https://doi.org/10.1080/10852352.2016.1198157

Gustavson, D. E., y Miyake, A. (2017). Academic procrastination and goal accomplishment: A combined experimental and individual differences investigation. Learning and Individual Differences, 54, 160-172. https://doi.org/10.1016/i.lindif.2017.01.010

Gustavson, D. E., Miyake, A., Hewitt, J. K., y Friedman, N. P. (2014). Genetic relations among procrastination, impulsivity, and goal-management ability: Implications for the evolutionary origin of procrastination. Psychological Science, 25(6), 1178-1188. https://doi.org/10.1177/0956797614526260

${ }^{*} \mathrm{Hajloo}, \mathrm{N}$. (2014). Relationships between self-efficacy, self-esteem and procrastination in undergraduate psychology students. Iranian Journal of Psychiatry and Behavioral Sciences, 8(3), 42-49. Retrieved from https://bit.ly/3xn2ZYB

Hamilton, W.K. (2018). MAJOR: Meta Analysis JamOvi R. For the jamovi project

Haney, P., y Durlak, J. A. (1998). Changing self-esteem in children and adolescents: A meta-analytical review. Journal of Clinical Child Psychology, 27(4), 423-433. https://doi.org/10.1207/s15374424jiccp2704 6

*Harrington, N. (2005). It's too difficult! Frustration intolerance beliefs and procrastination. Personality and Individual Differences, 39(5), 873-883. https://doi.org/10.1016/i.paid.2004.12.018

Hen, M., y Goroshit, M. (2014). Academic Procrastination, Emotional Intelligence, Academic Self-Efficacy, and GPA: A Comparison Between Students With and Without Learning Disabilities. Journal of Learning Disabilities, 47(2), 116124. https://doi.org/10.1177/0022219412439325 
Hidalgo-Fuentes, S., Martínez-Álvarez, I., y Sospedra-Baeza, M. J. (2021). Rendimiento académico en universitarios españoles: el papel de la personalidad y la procrastinación académica. European Journal of Education and Psychology, 14(1), 1-13. https://doi.org/10.32457/ejep.v14i1.1533

Higgins, J. P., y Thompson, S. G. (2002). Quantifying heterogeneity in a meta-analysis. Statistics in Medicine, 21(11), 1539-1558. https://doi.org/10.1002/sim.1186

Higgins, J. P., Thompson, S. G., Deeks, J. J., y Altman, D. G. (2003). Measuring inconsistency in meta-analyses. BMJ, 327(7414), 557-560. https://doi.org/10.1136/bmi.327.7414.557

Hunter, J. E., y Schmidt, F. L. 2004. Methods of meta-analysis: Correcting error and bias in research findings (2nd ed.). Sage Publications.

Janssen, J. (2015). Academic procrastination: Prevalence among high school and undergraduate student and relationship to academic achievement [Doctoral dissertation]. Georgia State University. Retrieved from https://bit.ly/3r5pi3R

*Kandemir, M., İlhan, T., Özpolat, A. R., y Palancı, M. (2014). Analysis of academic self-efficacy, self-esteem and coping with stress skills predictive power on academic procrastination. Educational Research and Reviews, 9(5), 146-152. https://doi.org/10.5897/err2014.1763

*Kınık, Ö., y Odacı, H. (2020). Effects of dysfunctional attitudes and depression on academic procrastination: does selfesteem have a mediating role?. British Journal of Guidance \& Counselling, 48(5), 638-649. https://doi.org/10.1080/03069885.2020.1780564

*Klassen, R. M., y Kuzucu, E. (2009). Academic procrastination and motivation of adolescents in Turkey. Educational Psychology, 29(1), 69-81. https://doi.org/10.1080/01443410802478622

*Klassen, R. M., Krawchuk, L. L., y Rajani, S. (2008). Academic procrastination of undergraduates: Low self-efficacy to self-regulate predicts higher levels of procrastination. Contemporary Educational Psychology, 33(4), 915-931. https://doi.org/10.1016/i.cedpsych.2007.07.001

*Klibert, J., Langhinrichsen-Rohling, J., Luna, A., y Robichaux, M. (2011). Suicide proneness in college students: Relationships with gender, procrastination, and achievement motivation. Death Studies, 35(7), 625-645. https://doi.org/10.1080/07481187.2011.553311

Klingsieck, K. B., Grund, A., Schmid, S., y Fries, S. (2013). Why students procrastinate: A qualitative approach. Journal of College Student Development, 54(4), 397-412. https://doi.org/10.1353/ csd.2013.0060

Kong, F., Ding, K., y Zhao, J. (2015). The relationships among gratitude, self-esteem, social support and life satisfaction among undergraduate students. Journal of Happiness Studies, 16(2), 477-489. https://doi.org/10.1007/s10902-0149519-2

Lay, C. H. (1986). At last, my research article on procrastination. Journal of Research in Personality, 20, 474-495. https://doi.org/10.1016/0092-6566(86)90127-3

Leary, M. R., y MacDonald, G. (2003). Individual differences in self-esteem: A review and theoretical integration. In M. R. Leary y J. P. Tangney (Eds.), Handbook of self and identity (pp. 401-418). Guilford Press.

Limone, P., Sinatra, M., Ceglie, F., y Monacis, L. (2020). Examining procrastination among university students through the lens of the self-regulated learning model. Behavioral Sciences, 10(12), 1-10. https://doi.org/10.3390/bs10120184

*Macher, D., Paechter, M., Papousek, I., y Ruggeri, K. (2012). Statistics anxiety, trait anxiety, learning behavior, and academic performance. European Journal of Psychology of Education, 27(4), 483-498. https://doi.org/10.1007/s10212-011-0090-5

Montes-Hidalgo, J., y Tomás-Sábado, J. (2016). Autoestima, resiliencia, locus de control y riesgo suicida en estudiantes de enfermería. Enfermeria Clinica, 26(3), 188-193. https://doi.org/10.1016/i.enfcli.2016.03.002

*Niu, Y., Jiang, N., y Jiang, X. (2021). Factors related to the resilience of Tibetan adolescent survivors ten years after the Yushu earthquake. International Journal of Disaster Risk Reduction, 65, 102554. https://doi.org/10.1016/j.ijdrr.2021.102554

Niveau, N., New, B., y Beaudoin, M. (2021). Self-esteem interventions in adults-a systematic review and meta-analysis. Journal of Research in Personality, 104131. https://doi.org/10.1016/i.jrp.2021.104131

*Özer, B. U., Saçkes, M., y Tuckman, B. W. (2013). Psychometric properties of the Tuckman Procrastination Scale in a Turkish sample. Psychological Reports, 113(3), 874-884. https://doi.org/10.2466/03.20.pr0.113x28z7

Povedano, A., Hendry, L. B., Ramos, M. J., y Varela, R. (2011). Victimización escolar: clima familiar, autoestima y satisfacción con la vida desde una perspectiva de género. Psychosocial Intervention, 20(1), 5-12. https://doi.org/10.5093/in2011v20n1a1

*Pychyl, T. A., Coplan, R. J., y Reid, P. A. (2002). Parenting and procrastination: gender differences in the relations between procrastination, parenting style and self-worth in early adolescence. Personality and Individual Differences, 33(2), 271-285. https://doi.org/10.1016/s0191-8869(01)00151-9

${ }^{*}$ Rebetez, M. M. L., Rochat, L., y Van der Linden, M. (2015). Cognitive, emotional, and motivational factors related to procrastination: A cluster analytic approach. Personality and Individual Differences, 76, 1-6. https://doi.org/10.1016/j.paid.2014.11.044

Rebetez, M. M. L., Rochat, L., Barsics, C., y Van der Linden, M. (2016). Procrastination as a self-regulation failure: The role of inhibition, negative affect, and gender. Personality and Individual Differences, 101, 435-439. https://doi.org/10.1016/j.paid.2016.06.049 
Robins, R. W., y Trzesniewski, K. H. (2005). Self-esteem development across the lifespan. Current Directions in Psychological Science, 14(3), 158-162. https://journals.sagepub.com/doi/abs/10.1111/j.0963-7214.2005.00353.x

Rosenberg, M. (1965). Society and the adolescent self image. Princeton University Press.

Rozental, A., Bennett, S., Forsström, D., Ebert, D. D., Shafran, R., Andersson, G., y Carlbring, P. (2018). Targeting procrastination using psychological treatments: A systematic review and meta-analysis. Frontiers in Psychology, 9 , 1588. https://doi.org/10.3389/fpsyg.2018.01588

San Martín, J. L., y Barra, E. (2013). Autoestima, apoyo social y satisfacción vital en adolescentes. Terapia Psicológica, 31(3), 287-291. https://doi.org/10.4067/S0718-48082013000300003

*Selçuk, Ş., Koçak, A., Mouratidis, A., Michou, A., y Sayıl, M. (2021). Procrastination, perceived maternal psychological control, and structure in math class: The intervening role of academic self-concept. Psychology in the Schools, 58(9), 1782-1798. https://doi.org/10.1002/pits.22542

*Sirois, F. M. (2004). Procrastination and counterfactual thinking: Avoiding what might have been. British Journal of Social Psychology, 43(2), 269-286. https://doi.org/10.1348/0144666041501660

Sirois, F. M., y Giguère, B. (2018). Giving in when feeling less good: Procrastination, action control, and social temptations. British Journal of Social Psychology, 57(2), 404-427. https://doi.org/10.1111/bjso.12243

Sirois, F. M, y Pychyl, T. A. (2013). Procrastination and the Priority of Short-Term Mood Regulation: Consequences for Future Self. Social and Personality Psychology Compass, 7(2), 115-127. https://doi.org/10.1111/spc3.12011

Sirois, F. M., y Pychyl, T. A. (2016). Procrastination, health, and well-being. Academic Press.

Sommer, L., y Haug, M. (2012). What Influences Implementation Intentions in an Academic Learning Context--The Roles of Goal Intentions, Procrastination, and Experience. International Journal of Higher Education, 1(1), 32-61. https://doi.org/10.5430/iihe.v1n1p32

Steel, P. (2007). The nature of procrastination: a meta-analytic and theoretical review of quintessential self-regulatory failure. Psychological Bulletin, 133(1), 65-94. https://doi.org/10.1037/0033-2909.133.1.65

Steel, P. (2011). The procrastination equation: How to stop putting things off and start getting stuff done. Pearson Education Limited.

Steel, P., y Klingsieck, K. B. (2016). Academic Procrastination: Psychological Antecedents Revisited. Australian Psychologist, 51(1), 36-46. https://doi.org/10.1111/ap.12173

Suurmond, R., van Rhee, H., y Hak, T. (2017). Introduction, comparison, and validation of Meta-Essentials: a free and simple tool for meta-analysis. Research synthesis methods, 8(4), 537-553. https://doi.org/10.1002/irsm.1260

*Tan, J. F., Ma, Z. W., y Li, X. T. (2015). Global self-esteem mediates the effect of general self-efficacy on Chinese undergraduates' general procrastination. Social Behavior and Personality: An International Journal, 43(8), 12651271. https://doi.org/10.2224/sbp.2015.43.8.1265

*Uribe, Y. C., Alegría, O. F., Shardin-Flores, N., y Luy-Montejo, C. A. (2020). Academic Procrastination, Self-Esteem and Self-Efficacy in University Students: Comparative Study in Two Peruvian Cities. International Journal of Criminology and Sociology, 9, 2474-2480. Retrieved from https://bit.ly/3r7FYHX

Wang, P., y Lei, L. (2021). How does problematic smartphone use impair adolescent self-esteem? A moderated mediation analysis. Current Psychology, 40(6), 2910-2916. https://doi.org/10.1007/s12144-019-00232-x

Yang, Z., Asbury, K., y Griffiths, M. D. (2019). An exploration of problematic smartphone use among Chinese university students: Associations with academic anxiety, academic procrastination, self-regulation and subjective wellbeing. International Journal of Mental Health and Addiction, 17(3), 596-614. https://doi.org/10.1007/s11469-018-9961-1

*Zhang, Y., Dong, S., Fang, W., Chai, X., Mei, J., y Fan, X. (2018). Self-efficacy for self-regulation and fear of failure as mediators between self-esteem and academic procrastination among undergraduates in health professions. Advances in Health Sciences Education, 23(4), 817-830. https://doi.org/10.1007/s10459-018-9832-3

Zhang, S., y Feng, T. (2019). Modeling Procrastination: Asymmetric Decisions to Act Between the Present and the Future. Journal of Experimental Psychology: General, 149(2), 311-322. https://doi.org/10.1037/xge0000643 